Pure \& App Z.Chem., Vo1.55, No.2, pp.229-236, 1983. Printed in Great Britain.
0033-4545/83/020229-08\$03.00/0

Pergamon Press Ltd.

()1983 IUPAC

\title{
APPLICATIONS OF MOLECULAR ORBITAL-VALENCE BOND THEORY IN CHEMISTRY
}

Nicolaos D. Epiotis

Department of Chemistry, University of Washington, Seattle, Washington 98195, USA.

Abstract--According to Molecular Orbital-Valence Bond (MOVB) theory, ground state organic molecules can be viewed as the result of "forbidden" union of two fragments. Consequently, the stability of a molecule is a function of the fragment excitation requirement for its formation and chemical stereoselection a manifestation of fragment excitation reduction. The pi electronic structure of benzene and the conformational isomerism of $\mathrm{N}_{2} \mathrm{H}_{4}$ and its derivatives are two simple illustrators of how and why MOVB theory has revolutionized our own thinking about the structure and reactivity of molecules.

MOVB Theory is the qualitative theory which stands at the same level of sophistication as the "best" ab initio polydeterminantal VB or MO computation, which has unparalleled conceptual clarity, and which can be equally well taught to chemistry beginners and to theoretical experts. The formal development and illustrative applications of MOVB theory were presented in a recent work. ${ }^{1}$ This lecture is an introduction to a second volume which contains applications of MOVB theory to different types of problems: organic and inorganic, ground state and excited state, thermodynamic and kinetic, etc. ${ }^{2}$ This yet unpublished volume defines what I call the "nightmare scenario of chemistry", i.e., it argues that our present understanding of chemistry at the electronic level is to a large extent illusory. In support of this proposition, I will discuss two entirely different problems with the first illustrating the conceptual advantage of MOVB theory over any M0-type theory and with the second illustrating the formal advantage of MOVB theory over the popular Hückel (HMO) theory (and any monodeterminantal MO or constrained VB theory). The conceptual clarity of MOVB theory is illustrated by reference to the problem of $\mathrm{N}_{2} \mathrm{X}_{4}$ and the formal "correctness" of MOVB theory by reference to the problem of benzene pi "aromaticity". In both cases, the emerging conclusions are nowhere to be fourd in the existing scientific literature. Thus, I argue that the fact that $\mathrm{N}_{2} \mathrm{H}_{4}$ is gauche rather than trans has nothing to do with the nitrogen lone pairs and that benzene is pi destabilized rather than pi stabilized! Both of these 
conclusions are the indirect result of a fundamental realization: most ground state molecules can be thought of as the products of "forbidden" unions of core and ligand fragments.

\section{A. $\mathrm{N}_{2} \mathrm{X}_{4}$ CONFORMAT IONAL ISOMERISM}

Like "aromaticity", hyperconjugation is nearly a half-century old idea. It refers to the "mechanism" by which the interaction of an electron pair bond with a vicinal doubly occupied, singly occupied, or vacant A0, or, with a vicinal electron pair bond confers stabilization to the total system in question. The original concept of hyperconjugation was founded on intuitive VB theory. Mulliken placed the idea of hyperconjugation on an MO theoretical basis in 1941. In recent times, the hyperconjugation argument has been enunciated in the language of Frontier Orbital (FO) Perturbation MO (PMO) theory. A number of investigators have made imaginative use of the concept of hyperconjugation in rationalizing and predicting chemical phenomena. ${ }^{3}$ After some time of intense fascination with these ideas and extensive exploration of their applicability, we began to realize that the successes of hyperconjugation were rivaled by failures for which no easy rationalization could be offered. A typical example is provided by the comparative study of conformational isomerism of $\mathrm{H}_{2} \mathrm{O}_{2}$ and $\mathrm{N}_{2} \mathrm{H}_{4}$. As first pointed out by Bauer and Yokozeki, ${ }^{4}$ the gauche preference of $\mathrm{H}_{2} \mathrm{O}_{2}$ seems to be augmented while the same preference of $\mathrm{N}_{2} \mathrm{H}_{4}$ is diminished upon replacement of the hydrogens by fluorines despite the fact that hyperconjugation ascribes the gauche preference of both $\mathrm{H}_{2} \mathrm{O}_{2}$ and $\mathrm{N}_{2} \mathrm{H}_{4}$ to the same stabilization "mechanism" and, thus, forecasts an identical response to substitution of the 1 igands or the core. In this section, I will demonstrate that $\mathrm{H}_{2} \mathrm{O}_{2}$ and $\mathrm{N}_{2} \mathrm{H}_{4}$ are qualitatively unrelated systems, the gauche preferences of which are due to entirely different factors. As a result, the effect of substituents on conformational isomerism is entirely different in the two systems. In other words, hyperconjugation works by accident in rationalizing the preferred conformation of $\mathrm{H}_{2} \mathrm{O}_{2}$ and $\mathrm{N}_{2} \mathrm{H}_{4}$ and it fails naturally in rationalizing the effects of substituents upon conformational preferences. Since $\mathrm{H}_{2} \mathrm{O}_{2}$ is gauche for the same reason that $\mathrm{H}_{2} \mathrm{O}$ and singlet $\mathrm{CH}_{2}$ are bent, ${ }^{1}$ our discussion is confined to $\mathrm{N}_{2} \mathrm{H}_{4}$ and derivatives.

The preferred geometry of $\mathrm{N}_{2} \mathrm{H}_{4}$ can be predicted (or, better, anticipated) by MOVB theory in a very simple and straightforward manner. Thus, one may consider three likely geometries, planar $(P)$, trans $(T)$, and gauche $(G)$ and compare their electronic structure by reference to the corresponding bond diagrams. These can be constructed by assuming a core-ligand dissection in which $\mathrm{N}_{2}$ plays the role of the core and $\mathrm{H}_{4}$ the role of the ligand fragment, developing the core and fragment symmetry orbitals according to well known perturbation theoretical procedures, and identifying the core-ligand bonds dictated by orbital symmetry. Now, as we have emphasized before, ${ }^{1}$ one cannot understand molecular stereochemistry unless the 
role of nonbonded interaction is properly appreciated. With this in mind, the symmetry orbitals of $\mathrm{H}_{4}, \sigma_{1}-\sigma_{4}$, of $\mathrm{N}_{2} \mathrm{H}_{4}$ are constructed recognizing that:

a) Geminal nonbonded overlap of the hydrogen $1 \mathrm{~s} A 0^{\prime}$ 's is very large. For example, using $r_{\mathrm{NN}}=1.47 \AA$ and $r_{\mathrm{NH}}=1.42 \AA$, it is computed to be 0.1542 in $\mathrm{P} \mathrm{N}_{2} \mathrm{H}_{4}$.

b) Vicinal nonbonded overlap of the hydrogen $1 \mathrm{~s} A 0^{\prime} \mathrm{s}$ is much smaller but far from negligible. For example, it is computed to be 0.0338 in $\mathrm{P} \mathrm{N}_{2} \mathrm{H}_{4}$.

Statements (a) and (b) are strictly valid whenever the core is made up of first row atoms. When the core is made up of second, third, etc., row atoms, the increasing core atom-1igand atom bond lengths cause the ligands to move further away from each other and nonbonded ligand A0 overlap tends to become negligible. These above considerations lead to the conclusion that the final symmetry MO's of $\mathrm{H}_{4}$ will separate into two bands so that electron excitation from $\sigma_{1}$ or $\sigma_{2}$ to $\sigma_{3}$ or $\sigma_{4}$ will always be costly and the corresponding deexcitation beneficial regardless of geometry. The qualitative energy levels of $H_{4}$ appropriate to $P, T$ and $G$ $\mathrm{N}_{2} \mathrm{H}_{4}$ would be radically different had we assumed that nonbonded overlap of the hydrogen $1 \mathrm{~s}$ AO's is zero. In such an event, excitation or deexcitation could no longer be defined simply because all four MO's would be degenerate. We will see that assumption of one or the other $\mathrm{H}_{4}$ MO patterns makes a "night-day" difference insofar as the final prediction of the preferred geometry of $\mathrm{N}_{2} \mathrm{H}_{4}$ is concerned.

The bond diagrams for $P, T$, and $\mathrm{GN}_{2} \mathrm{H}_{4}$ are shown in Figure 1 . We note the following:

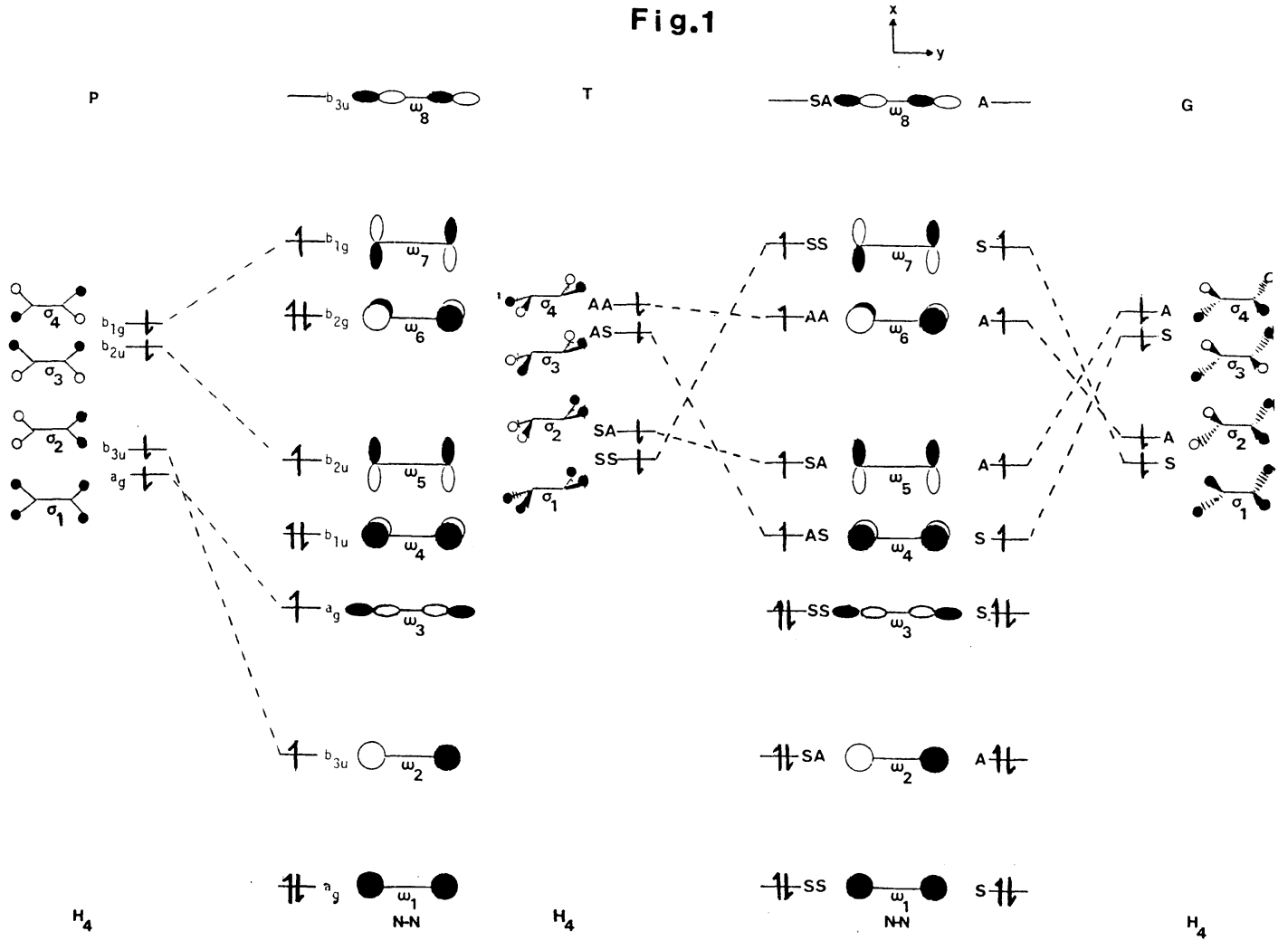


a) In comparing $P$ and $T$, we first recognize that the former is the maximum overlap form. Hence, a dagger must be affixed to whatever label is appropriate for $T$. The transition $\mathrm{P} \rightarrow \mathrm{T}$ is accompanied by $\mathrm{U} \rightarrow \mathrm{H}^{\neq}$rebonding. In more descriptive language, we can say that it is accompanied by core deexcitation of the $\omega_{6} \rightarrow \omega_{2}$ type. Since the energy separation of the $\omega_{2}$ and $\omega_{6}$ orbitals is very large, we expect that deexcitation will dominate loss of spatial overlap and $T$ will attain lower energy than $P$.

b) In comparing $T$ and $G$, we recognize that the former is the maximum overlap form. The transition $T \rightarrow G$ is again accompanied by $U \rightarrow H^{\ddagger}$ rebonding. In more descriptive terms, we can say that it is accompanied by core plus 1 igand deexcitation of the $\sigma_{4} \rightarrow \sigma_{2}$ and $\omega_{6} \rightarrow \omega_{5}$ type. Since the energy separation of $\sigma_{4}$ and $\sigma_{2}$ as well as the energy separation of $\omega_{6}$ and $\omega_{5}$ are large we expect again that deexcitation will dominate the loss of spatial overlap and G will attain lower energy than $T$. Note that, if $\sigma_{1}$ to $\sigma_{4}$ were degenerate, the rebonding would be simply of the $N \rightarrow N^{\prime}$ type, i.e., rebonding accompanied by bond weakéning due to loss of spatial overlap without any deexcitation benefit. In this case, T would end up being energetically more favorable than $G$. Since whether $\sigma_{2}$ and $\sigma_{4}$ are degenerate or not depends on the strength of geminal nonbonded interaction, we can see that two different predictions can be arrived at depending on whether geminal nonbonded overlap is deemed important or not: If geminal nonbonded interaction is appreciable, $\sigma_{2}$ and $\sigma_{4}$ will be substantially split in energy, the $T \rightarrow G$ transformation will be accompanied by $U \rightarrow H^{\neq}$rebonding, and $G$ will be the lowest energy form. By contrast, if geminal nonbonded interaction is negligible, $\sigma_{1}$ to $\sigma_{4}$ will be degenerate, the $T \rightarrow G$ transformation will be accompanied by $N \rightarrow N$ ' rebonding and $T$ will be the lowest energy form. Since geminal nonbonded overlap is actually very large, the first prediction is the correct one.

Once we have properly understood why $\mathrm{N}_{2} \mathrm{H}_{4}$ is gauche, the reason for the fact that $\mathrm{N}_{2} \mathrm{~F}_{4}$ shows no comparable preference for the gauche geometry becomes self evident. Specifically, because of the smaller spatial extension of the fluorine valence AO's, geminal nonbonded overlap between $\mathrm{F}$ and $\mathrm{F}$ in $\mathrm{N}_{2} \mathrm{~F}_{4}$ is much smaller than between $\mathrm{H}$ and $\mathrm{H}$ in $\mathrm{N}_{2} \mathrm{H}_{4}$. As a result, the motivation for the $T \rightarrow G$ transformation largely ceases to exist and $\mathrm{N}_{2} \mathrm{~F}_{4}$ tends to adopt a $T$, rather than a $G$, geometry for reasons explained above.

Finally, consider the molecule $\mathrm{AH}_{n}$ in which $A$ is a first row atom. Replacement of $A$ by a second row atom of the same column of the Periodic Table will cause an increase of the H---H distance, $r_{H H}$, and a very significant reduction of nonbonded AO overlap. This suggests that, upon replacing $\mathrm{N}$ by $\mathrm{P}$ in $\mathrm{N}_{2} \mathrm{X}_{4}$, we must observe a dramatic reduction of the energy difference between the $G$ and the $T$ form and even a reversal of its sign. That is to say, we expect that the diminution of the geminal nonbonded interaction will tend to convert the rebonding accompanying the $T \rightarrow G$ transformation from $U \rightarrow H^{\neq}$to $N \rightarrow N^{\prime}$ and, thus, reverse the order of relative stability of $T$ and $G$. The trend will become even more pronounced as $P$ 
is replaced by As. The available experimental results leave no doubt that, as the covalent radius of $A$ increases, the trans becomes increasingly more stable relative to the gauche conformer. This trend is exactly opposite to the one encountered in ethane-like $A_{2} X_{6}$ molecules and it has been noted by several authors. 5

\section{B. WHY PI BENZENE IS NOT WHAT CHEMISTS THINK IT IS.}

Hückel $M O$ (HMO) theory predicts that pi benzene is more stable than three pi ethylenes. This conclusion seems to be compatible with the fact that the heat of hydrogenation of benzene is much less than the heat of hydrogenation of three cyclohexenes and the known unwillingness of benzene to undergo addition reactions, opting for aromatic substitution instead. These data have prompted an on-going preoccupation with "resonance energies", "aromaticity", and the like. In this section, we suggest that, while the experimental facts are indisputable, the concepts which chemists have devised over more than a century are most likely erroneous and that benzene is pi destabilized yet "aromatic".

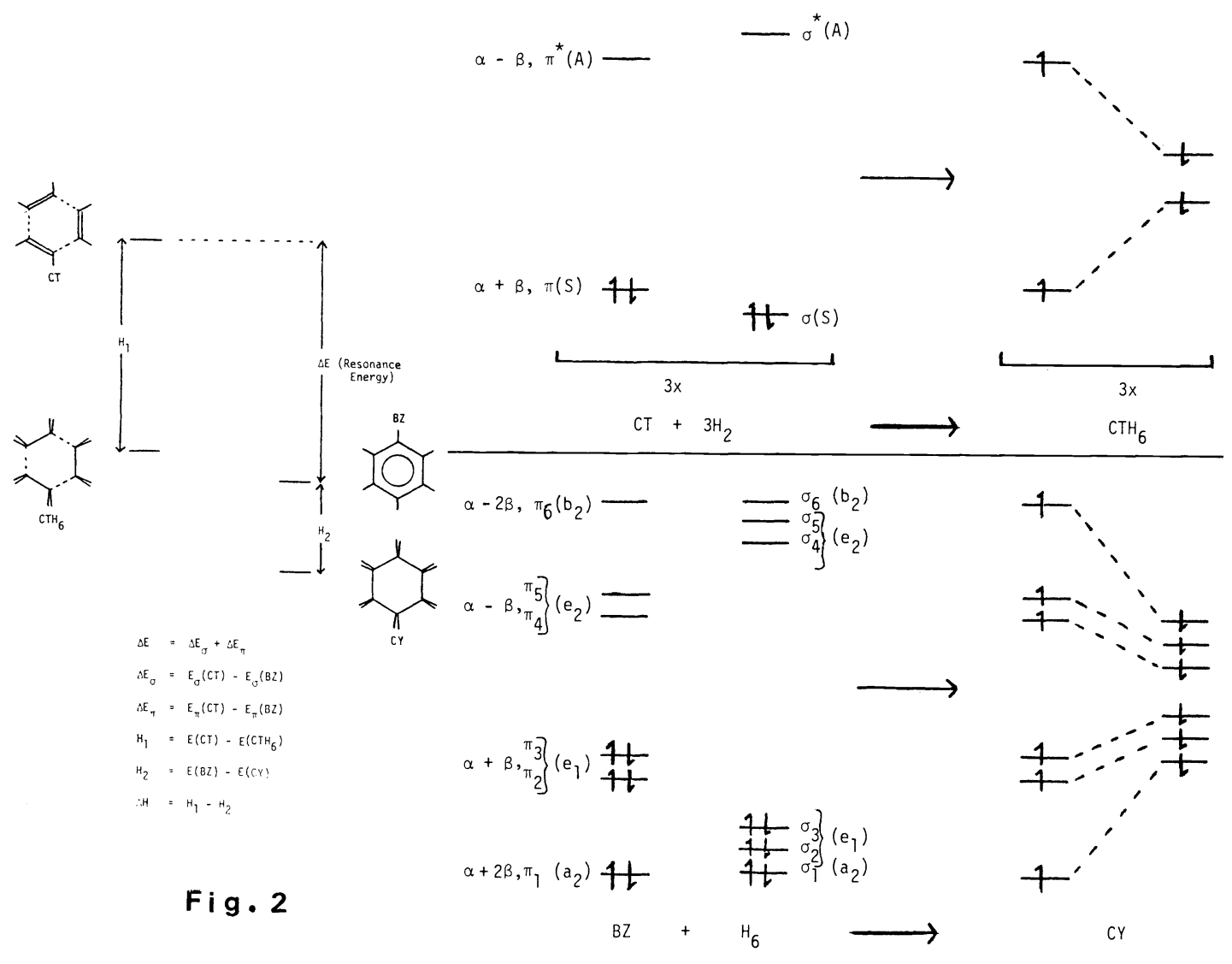

F i g . 3 
There are two equivalent ways of explaining why the heat of hydrogenation of benzene (BZ) is much less negative than that of 1,3,5-cyclohexatriene (CT): One is to compare CT and $B Z$ directly and another is to examine the difference between the $\mathrm{CT} \rightarrow \mathrm{CTH}_{6} \rightarrow \mathrm{CY}$ and the $B Z \rightarrow C Y$ transformations depicted in Figure 2. That is to say, we can describe a clockwise $(\mathrm{CT} \rightarrow \mathrm{BZ})$ and counterclockwise $\left(\mathrm{CT} \rightarrow \mathrm{CTH}_{6} \rightarrow \mathrm{CY} \rightarrow \mathrm{BZ}\right)$ thermochemical cycle realizing that the end result is always the same but the associated explanations are not necessarily identical. In one case, in travelling from $C T$ to $B Z$, there is sigma bond formation accompanied by "aromatic" pi delocalization. Now, such delocalization lowers the one-electron energy of a system, because of the interaction of "covalent" and "ionic" CW's, at the expense of strong interelectronic repulsion, the characteristic attribute of "ionic" CW's. Hence, in comparing $C T$ and $B Z$, one must consider one-electron and two-electron effects while recognizing that HMO theory describes overdelocalization because of the neglect of electronelectron interaction. By contrast, in travelling from CT to $\mathrm{CTH}_{6}$ to $\mathrm{CY}$ to $\mathrm{BZ}$, one no longer has to deal with "aromatic" pi delocalization. Rather, attention is now focused exclusively on the energy for converting $\mathrm{C}-\mathrm{C}$ pi to $\mathrm{C}-\mathrm{H}$ sigma bonds, i.e., the $\mathrm{CT} \rightarrow \mathrm{CHT}_{6}$ and $\mathrm{BZ} \rightarrow \mathrm{CY}$ transformations, as well as the energetics of sigma bond formation between saturated fragments having doubly occupied orbitals which can only engender strong interfragmental overlap repulsion, i.e., the $\mathrm{CTH}_{6} \rightarrow \mathrm{CY}$ transformation. Now, addition of $3 \mathrm{H}_{2}$ to $\mathrm{CT}$ and $\mathrm{BZ}$ requires intrafragmental excitations which cause similar readjustments of the relative weights of the "ionic" and "covalent" CW's. Furthermore, the transformation of CTH $_{6}$ to CY involves formation of relatively localized sigma bonds. As a result, one may assume that the $\mathrm{CT} \rightarrow \mathrm{CTH}_{6} \rightarrow \mathrm{CY} \rightarrow \mathrm{BZ}$ thermochemistry is determined, in a qualitative sense, by only oneelectron effects because the change of interelectronic repulsion accompanying the $\mathrm{CT} \rightarrow \mathrm{CTH}_{6}$ tends to cancel that accompanying the $\mathrm{CY} \rightarrow \mathrm{BZ}$ transformation while the $\mathrm{CTH}_{6} \rightarrow \mathrm{CY}$ conversion involves no appreciable change of electron repulsion due to bond localization. Recognizing that the $\mathrm{CT} \rightarrow \mathrm{BZ}$ will be a more exothermic process than the $\mathrm{CTH}_{6} \rightarrow \mathrm{CY}$ transformation, it is immediately evident that the problem of benzene "aromaticity" can be considered solved if one can explain why $\Delta H$ is positive, assuming that interelectronic repulsion effects are unimportant, and determine whether $\Delta \mathrm{E}_{\pi}$ is indeed positive, as predicted by HMO theory, or actually negative as a result of bielectronic destabilization dominating monoelectronic stabilization of the cyclic pi system of benzene. We now show that $\Delta H$ and $\Delta E$ have opposite signs and that $\Delta \mathrm{E}_{\pi}$ has probably the same sign as $\Delta \mathrm{E}$. In other words, we argue that the correct interpretation of benzene "aromaticity" is tantamount to a proper rationalization of the sign of either $\Delta \mathrm{H}$ or $\Delta \mathrm{E}$ and that $\Delta \mathrm{E}_{\pi}$ actually leads to the prediction that benzene is pi destabilized. Thus, the fact that benzene resists addition, while an olefin readily undergoes such a reaction, can be explained in two different, yet equivalent ways, with the $\Delta H$ explanation being the more physically meaningful one as it directly links the pi 
electronic structure of two reactants to their differing ability to form "new" sigma bonds to replace the "old" pi bonds. In what follows, we make consistent usage of the definitions spelled out in Figure 2.

In general, $H_{i}$ reflects two factors:

a. The energy required for preparation of the reactants for bonding, i.e., the requisite reactant excitation energies.

b. The energy payback due to bond formation.

Since we make $\mathrm{C}-\mathrm{H}$ bonds in both $\mathrm{CT}$ and $\mathrm{BZ}$, the $\mathrm{AO}$ resonance integrals describing bond formation are kept constant. Thus, the only difference between $\mathrm{CT}$ and $\mathrm{BZ}$ with respect to reaction with $\mathrm{H}_{6}$ is due to the differing excitation energies. The bond diagrams which show explicitly how $\mathrm{CTH}_{6}$ is formed by adding $\mathrm{CT}$ and $\mathrm{H}_{6}$ and how $\mathrm{CY}$ arises from the union of $\mathrm{BZ}$ and $\mathrm{H}_{6}$ are shown in Figure 3. Both $\mathrm{CTH}_{6}$ and $\mathrm{CY}$ can be regarded as the products of the "forbidden" unions of $\mathrm{CT}$ plus $3 \mathrm{H}_{2}$ and $\mathrm{BZ}$ plus $3 \mathrm{H}_{2}$, respectively. It is immediately evident that there is a greater excitation requirement in the case of BZ than in the case of CT. Hence, $\Delta H$ is predicted to be positive. Since $\Delta H$ is a measure of relative reaction exothermicity, we say that CT is a hyperdesmic and BZ is a hypodesmic molecule, in a relative sense.

Why does HMO theory predict that pi benzene is stable with respect to three pi ethylenes? We immediately recognize that, if we view each of CT and BZ as a composite of three $H C=C H$ fragments $\left(F_{1}, F_{2}, F_{3}\right)$, there exists a set of purely "ionic" charge transfer MOVB CW's which "contain" VB CW's that can contribute to the VB wavefunction of BZ but not to that of CT, e.g., $\Phi_{1}$ and $\Phi_{2}$ shown below. "Excusing" interelectronic repulsion at the level of HMO theory makes $\Phi_{1}$ and $\Phi_{2}$ unduly important and causes BZ to be more stable than CT.

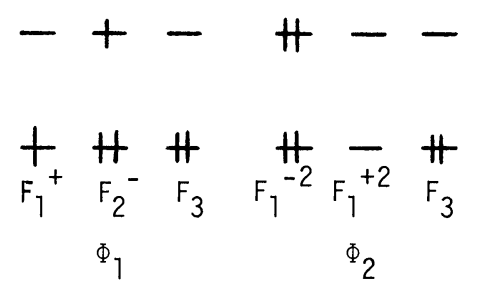

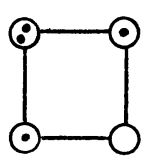

$x_{1}$

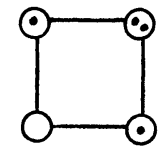

$x_{2}$

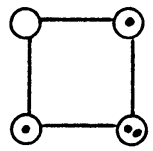

$x_{3}$

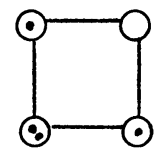

$x_{4}$

A related example drawn from our previous work ${ }^{1}$ is the constitution of the VB wavefunctions of pi cyclobutadiene with noninteracting pi bonds $\left(\mathrm{CB}^{\star}\right)$ and Möbius pi cyclobutadiene (MCB), two hypothetical systems analogous to CT and BZ. According to VB theory, and in complete analogy to MOVB theory, the advantage of MCB over $C B^{\star}$ lies in four unique "ionic" VB CW's, $x_{1}-x_{4}$, which contribute to the wavefunction of the former but not the latter system. Once again, neglect of interelectronic repulsion at the level of HMO theory causes these unique "ionic" CW's to achieve undue prominence and stabilize the "allowed" or "aromatic" MCB 
complex relative to the "nonaromatic" $C B^{*}$ more than it "deserves".

It is then evident that we cannot neglect interelectronic repulsion because we deal with interbond excitations (charge transfer) in the case of BZ which can only occur at the expense of severe interelectronic repulsions and which, if neglected, would lead one to believe that $B Z$ is more stable than CT while, if included, they can lead to exactly the opposite conclusion, i.e., that $\Delta E_{\pi}$ is negative. That pi CT is more stable than pi BZ has been implicitly suggested more than a half century ago when Eyring used the Diatomics In Molecules method ${ }^{6}$ to show that $3 A_{2}$ is more stable than hexagonal $A_{6}$ provided that $A$ is a strong overlap binder. ${ }^{2} A_{6}$ can become more stable than $3 A_{2}$ only when $A$ is a weak overlap binder (e.g., Li) in which case the greater stability of hexagonal $A_{6}$ is due to the fact that such atoms bind to a large extent via "classical" coulomb interactions in the absence of interatomic charge transfer. ${ }^{7}$

\section{REFERENCES}

1. N. D. Epiotis, J. R. Larson, and H. Eaton, "Unified Valence Bond Theory of Electronic Structure", in Lecture Notes in Chemistry, Vol. 29, Springer-Verlag, Heidelberg, 1982.

2. N. D. Epiotis, "The New Valence Bond World of Chemistry", to be published.

3. For leading references, see ref. 1 .

4. A. Yokozeki and S. H. Bauer, Top. Curr. Chem. 53, 71 (1974).

5. A. H. Cowley, M. J. S. Dewar, D. W. Goodman, and M. C. Padolina, J. Am. Chem. Soc. 96, 2648 (1974).

6. S. Glasstone, K. J. Laidler, and H. Eyring, "The Theory of Rate Processes", McGraw-Hill, New York, 1941. For a clear presentation of the DIM theory of $A_{2}$ and $A_{6}$, see:

C. Sandorfy, "Electronic Spectra and Quantum Chemistry", Prentice-Hal1, Englewood Cliffs, N.J., 1964. For an important parallel DIM and MO study of the degenerate isomerization of $3 \mathrm{H}_{2}$, see: D. A. Dixon, R. M. Stevens, and D. R. Herschbach, Faraday Discussion of Chem. Soc. 62, 110 (1977).

7. Of course, in order to explain why $\Delta H$ is positive, we had to first recognize that the $\mathrm{CT} \rightarrow \mathrm{BZ}$ is more exothermic than the $\mathrm{CTH}_{6} \rightarrow \mathrm{CY}$ transformation because the former engenders less interfragmental overlap repulsion than the latter as the pi electrons of CT are delocalized more than the $\mathrm{C}-\mathrm{H}$ sigma electrons of $\mathrm{CTH}_{6}$. If the energy changes accompanying these two transformations are symbolized by $\Delta E$ and $\Delta E^{\prime}$, respectively, the $\Delta H$ explanation is entirely equivalent to the $\Delta E-\Delta E^{\prime}$ explanation but more appealing as it directly illuminates the source of the reactivity differences between olefinic and "aromatic" systems. 